\title{
Chemical composition of UV-bright star ZNG 4 in the globular cluster M $13^{\star, \star \star}$
}

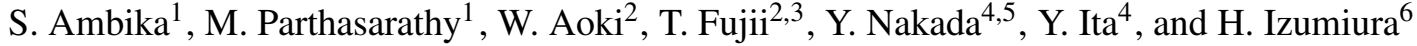 \\ 1 Indian Institute of Astrophysics, Koramangala, Bangalore, 560034, India \\ 2 National Astronomical Observatory, Mitaka, Tokyo 181-8588, Japan \\ 3 Faculty of Science, Kagoshima University, 1-21-35 Korimoto, Kagoshima 890-0065, Japan \\ ${ }^{4}$ Institute of Astronomy, The University of Tokyo, Mitaka, Tokyo, 181-0015, Japan \\ 5 Kiso Observatory, Institute of Astronomy, University of Tokyo, Mitake, Kiso, Nagano 397-0101, Japan \\ ${ }^{6}$ Okayama Astrophysical Observatory, National Astronomical Observatory, Kamogata, Okayama 719-0232, Japan
}

Received 20 May 2003 / Accepted 18 November 2003

\begin{abstract}
We present a detailed model-atmosphere analysis of ZNG 4, a UV-bright star in the globular cluster M 13. From the analysis of a high resolution $(R \approx 45000)$ spectrum of the object, we derive the atmospheric parameters to be $T_{\text {eff }}=$ $8500 \pm 250 \mathrm{~K}, \log g=2.5 \pm 0.5$ and $[\mathrm{Fe} / \mathrm{H}]=-1.5$. Except for magnesium, chromium and strontium, all other even $Z$ elements are enhanced with titanium and calcium being overabundant by a factor of $0.8 \mathrm{dex}$. Sodium is enhanced by a factor of $0.2 \mathrm{dex}$. The luminosity of ZNG 4 and its position in the color-magnitude diagram of the cluster indicate that it is a Supra Horizontal Branch (SHB) (post-HB) star. The underabundance of He and overabundances of Ca, Ti, Sc and Ba in the photosphere of ZNG 4 indicate that diffusion and radiative levitation of elements may be in operation in M 13 post-HB stars even at $T_{\text {eff }}$ of $8500 \mathrm{~K}$. Detailed and more accurate abundance analysis of post-HB stars in several globular clusters is needed to further understand their abundance anomalies.
\end{abstract}

Key words. stars: abundances - stars: evolution - stars: Population II - stars: horizontal-branch Galaxy: globular cluster: individual: M 13 - stars: individual: ZNG 4

\section{Introduction}

The term "UV-bright stars" was introduced by Zinn et al. (1972) for stars in globular clusters that lie above the horizontal branch (HB) and are bluer than red giants. The name resulted from the fact that, in the $U$ band, these stars were brighter than all other cluster stars. Further investigations showed that this group of stars consist of blue horizontal branch (BHB) stars, supra horizontal branch stars (SHB), post asymptotic giant branch stars (post-AGB), post-early AGB (P-EAGB) stars and AGB-manque stars (de Boer 1985, 1987; Sweigart et al. 1974; Brocato et al. 1990; Dorman et al. 1993; Gonzalez \& Wallerstein 1994).

To derive the chemical composition of UV-bright stars in globular clusters and to understand their evolutionary stages, we started a program to obtain high resolution spectra of these objects in selected globular clusters with the High Dispersion Spectrograph (HDS, Noguchi et al. 2002) of the $8.2 \mathrm{~m}$ Subaru

Send offprint requests to: $\mathrm{S}$. Ambika, e-mail: ambika@iiap.ernet.in

* Based on observations obtained with the Subaru $8.2 \mathrm{~m}$ Telescope, which is operated by the National Astronomical Observatory of Japan.

$\star \star$ Table 3 is only available in electronic form at http://www.edpsciences.org
Telescope. We selected a few UV-bright stars in the globular cluster M 13 from the papers of Zinn et al. (1972) and Harris et al. (1983) to derive their chemical composition. In this paper we report the analysis of a high resolution spectrum of the UV-bright star ZNG 4 (RA $\left(16^{\mathrm{h}} 41^{\mathrm{m}} 37^{\mathrm{s}} .528\right)$ and Dec $\left(+36^{\circ} 30^{\prime} 43.86^{\prime \prime}\right)(2000)$ ) (Zinn et al. 1972) in M 13 as the first target of our program.

M 13 (NGC 6205) is a nearby wellstudied globular cluster with a distance modulus of $(m-M)_{0}=14$ m. 42 and metallicity of $[\mathrm{Fe} / \mathrm{H}]=-1.51$ (Kraft \& Ivans 2003). The position of ZNG 4 in the color-magnitude diagram of M 13 (Paltrinieri et al. 1998) is shown in Fig. 1. Many of the globular clusters show a prominent gap in the blue tail of the $\mathrm{HB}$, which is presumed to be due to differential mass loss on the Red Giant Branch (RGB). In M 13 it is observed at $T_{\text {eff }}=10000 \mathrm{~K}$ (Ferraro et al. 1997). High resolution spectroscopic studies of M 13 BHB stars lying on either side of the gap were carried out by Peterson et al. $(1983,1995)$ and Behr et al. (1999, 2000a). They found anomalous photospheric abundances in BHB stars. These photospheric anomalies are most likely due to diffusion - the gravitational settling of helium and radiative levitation of the metal atoms in the stable atmosphere of hot stars. They found variations in the photospheric abundances and rotational velocities of BHB stars as a function of their effective temperatures. 


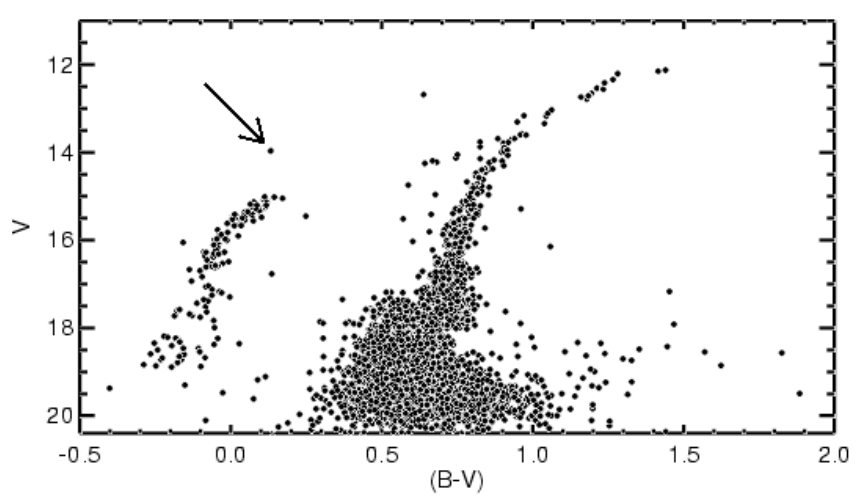

Fig. 1. Color magnitude diagram (CMD) of globular cluster M 13 obtained by Paltrinieri et al. (1998). The arrow indicates the position of ZNG 4 in the CMD.

\section{Observations}

We have obtained a high resolution $\left(\frac{\lambda}{\Delta \lambda} \approx 45000\right)$ spectrum of ZNG 4 on 15th (UT: 14h 45m) April 2001 with the Subaru/HDS. The spectrum covering the wavelength range $4142 \AA-6814 \AA$ was obtained in an exposure time of $20 \mathrm{~min}$. There was no moon light problem during the observations and the sky background in the data was close to zero. We neglected the sky background in our data reduction.

The data was bias-subtracted, trimmed, flat-fielded to remove pixel to pixel variations, converted to a one-dimensional spectrum, and normalized to the continuum using standard CCD data reduction package (NOAO IRAF). The spectrum has an average signal to noise ratio of 35 . The reference spectrum of thorium-argon was used for the wavelength calibration.

The various orders in our echelle spectrum of ZNG 4 have well defined continuum and the normalization of the continuum was carried out using the IRAF echelle spectra reduction programs. The continuum level in the adjacent echelle orders to those containing the Balmer lines was useful in defining the continuum in the Balmer line regions and the profiles were normalized with a polynomial fit.

\section{Analysis}

The spectral lines were identified using Moore's atomic multiplet table (1945). Equivalent widths of the absorption lines were measured using the routines available in the SPLOT package of IRAF. The equivalent widths were measured by Gaussian fitting to the observed profiles (and a multiple Gaussian fit to the blended lines such as the Mg II lines at $4481 \AA$ ) and are given in Table 3 .

\subsection{Radial velocity}

The radial velocity of ZNG 4 was derived from the wavelength shifts of many absorption lines. The average heliocentric velocity is found to be $V_{\mathrm{r}}=-257.56 \pm 1.08 \mathrm{~km} \mathrm{~s}^{-1}$ which is in agreement with the value derived by Zinn (1974) $\left(-253 \mathrm{~km} \mathrm{~s}^{-1}\right)$. It is also in agreement with the heliocentric velocities of M 13 BHB stars derived by Behr et al. (1999) and Moehler et al. (2003).

\subsection{Atmospheric parameters}

For the initial estimate of effective temperature, we looked for the published CCD photometry of the star. Recent CCD photometry of M 13 was carried out by Rey et al. (2001). However the ZNG 4 area of the cluster was not included in their observations (Rey, private communication). We used the published CCD photometry of ZNG 4 by Paltrinieri et al. (1998), who give, $B=14.096$ and $V=13.964$. $(B-V)=0.132$ and $E(B-$ $V)=0.02\left(\right.$ Kraft $\&$ Ivans 2003) will yield $(B-V)_{0}=0.112$ which corresponds to $T_{\text {eff }}=8373 \mathrm{~K}$ (Flower 1996). However, the $(B-V)_{0}$ and $T_{\text {eff }}$ calibration given by Flower (1996) is for Population I stars.

For our analysis, excitation potential and oscillator strengths of the lines were taken from the Vienna Atomic Line Database (http://www.astro.univie.ac. at/ vald/). We employed the latest (2002) version of MOOG, an LTE stellar line analysis program (Sneden 1973) and Kurucz (1993) grid of ATLAS models. MOOG has been used successfully in the analysis of the spectra of warmer stars with $T_{\text {eff }}=7900 \mathrm{~K}$ (Preston \& Sneden 2000).

We have also analyzed the spectra using the Kurucz WIDTH program (Kurucz CDROM 13, 1993) for verification. We used the line list obtained using version 43 of the Synspec code of Hubeny and Lanz which is distributed as part of their TLUSTY model atmosphere program. (http://tlusty. gsfc.nasa.gov/Synspec43/synspec-line.html) and also the information from the Kurucz linelist (http:// kurucz.harvard. edu/linelists.html).

The value of effective temperature was obtained by the method of excitation balance, forcing the slope of abundances from Fe I lines versus excitation potential to be zero. The surface gravity was then set by ionization- equilibrium, forcing abundances obtained from neutral (Fe I) and ionized (Fe II) species to be equal. The microturbulent velocity was estimated by demanding that there should be no dependence of the $\mathrm{Fe} I$ abundance upon equivalent widths of $\mathrm{Fe} I$ lines.

The plots of the abundances versus excitation potentials and abundances versus equivalent widths in the case of $\mathrm{Fe} \mathrm{I}$ and Fe II lines are shown in Fig. 2. Such plots were made by varying the $T_{\text {eff }}, \log g$ and $V_{\mathrm{t}}$ in steps of $250 \mathrm{~K}, 0.5$ and $0.5 \mathrm{~km} \mathrm{~s}^{-1}$ respectively to estimate the uncertainties in these parameters.

From our analysis, we find that $T_{\text {eff }}=8500 \mathrm{~K}, \log g=2.5$ and $V_{\mathrm{t}}=2.5 \mathrm{~km} \mathrm{~s}^{-1}$ fit the data best (Fig. 2). From the abovementioned method of analysis we find the uncertainties in $T_{\text {eff }}$ to be $250 \mathrm{~K}, \log g=0.5$ dex and $V_{\mathrm{t}}=0.5 \mathrm{~km} \mathrm{~s}^{-1}$. Uncertainties in derived abundances as a result of errors in the determination of the parameters and errors in the measurements of equivalent widths are found to be of the order of 0.2 dex.

Using the derived atmospheric parameters and abundances, a synthetic spectrum was generated and plotted over the observed spectrum for verification. The observed and synthetic spectra were found to match well with the above mentioned atmospheric parameters and the final abundances are given in Tables 1 and 3. A region of the observed and synthetic spectrum is shown in Fig. 3. The abundances derived using MOOG (Tables 1 and 3) are in good agreement with the abundances derived using the WIDTH (Table 1). 

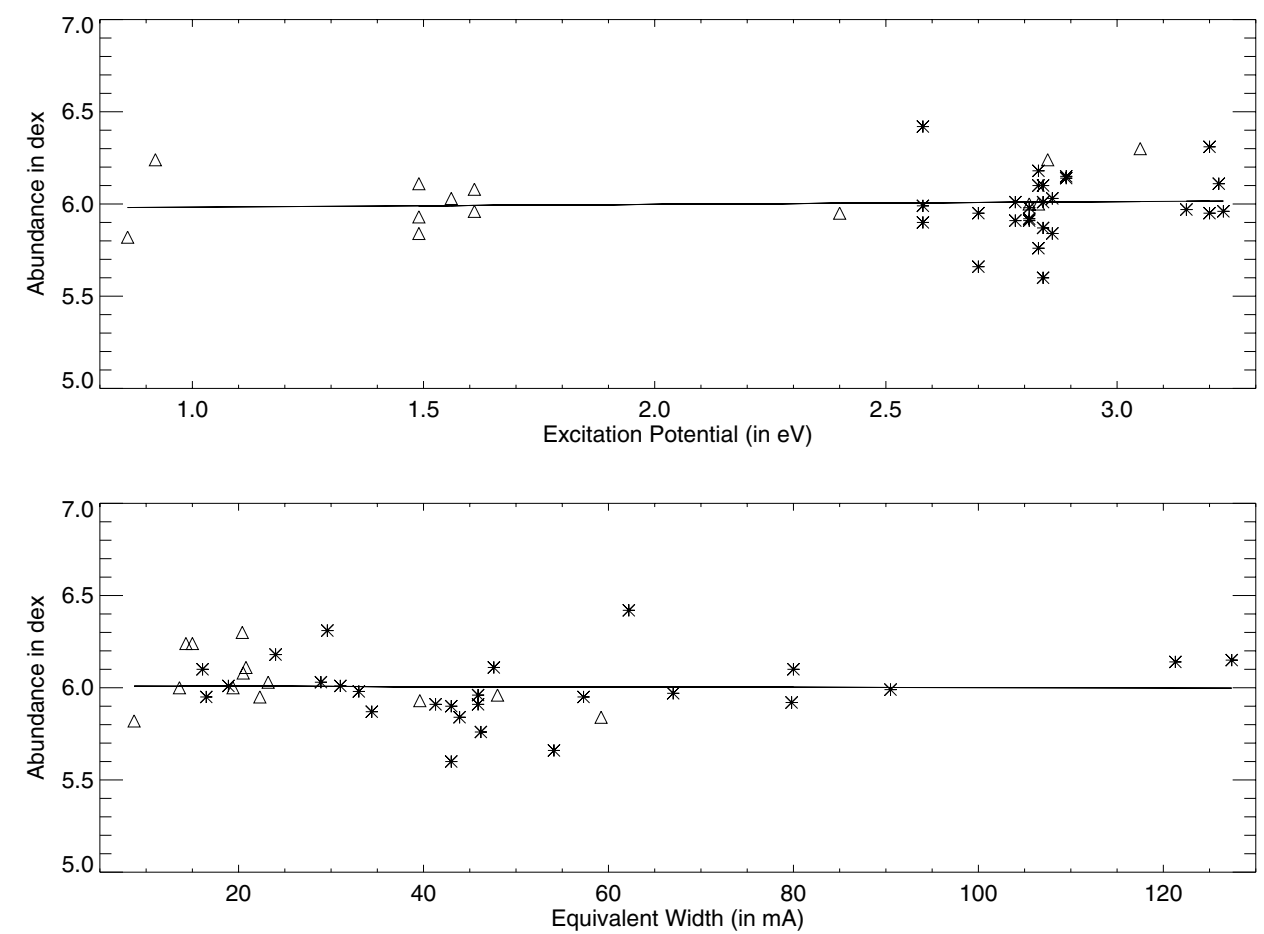

Fig. 2. Top figure is the plot of the abundances from Fe lines versus excitation potential of the lines. Figure at the bottom is the plot of the abundances from Fe lines versus their equivalent widths. Triangles represent the Fe I lines and stars denote the Fe II lines.

Table 1. Chemical composition of ZNG 4 in M 13.

\begin{tabular}{|c|c|c|c|c|c|c|}
\hline \multirow[t]{2}{*}{ Element } & \multirow[t]{2}{*}{ No. of lines } & \multicolumn{2}{|c|}{$\begin{array}{c}T_{\text {eff }}=8500 \mathrm{~K}, \log g=2.5 \\
V_{\mathrm{t}}=2 \mathrm{~km} \mathrm{~s}^{-1} \text { and } \\
{[\mathrm{Fe} / \mathrm{H}]=-1.5} \\
\text { MOOG }\end{array}$} & \multicolumn{2}{|c|}{$\begin{array}{c}T_{\text {eff }}=8500 \mathrm{~K}, \log g=2.5 \\
V_{\mathrm{t}}=2 \mathrm{~km} \mathrm{~s}^{-1} \text { and } \\
{[\mathrm{Fe} / \mathrm{H}]=-1.5} \\
\text { WIDTH }\end{array}$} & \multirow{2}{*}{$\begin{array}{c}T_{\text {eff }}=8750 \mathrm{~K}, \log g=2.0 \\
V_{\mathrm{t}}=2 \mathrm{~km} \mathrm{~s}^{-1} \text { and } \\
{[\mathrm{Fe} / \mathrm{H}]=-1.5} \\
\text { MOOG } \\
\mathrm{X} / \mathrm{H}] \pm \sigma\end{array}$} \\
\hline & & {$[\mathrm{X} / \mathrm{H}] \pm \sigma$} & {$[\mathrm{Ele} / \mathrm{Fe}]$} & {$[\mathrm{X} / \mathrm{H}] \pm \sigma$} & [Ele/Fe] & \\
\hline $\mathrm{He} \mathrm{I}$ & 1 & $-0.55 \pm 0.20$ & & $-0.68 \pm 0.20$ & & $-1.13 \pm 0.31$ \\
\hline $\mathrm{Na} \mathrm{I}$ & 2 & $-1.28 \pm 0.10$ & +0.21 & $-1.30 \pm 0.10$ & +0.20 & $-0.79 \pm 0.11$ \\
\hline Mg I & 3 & $-1.46 \pm 0.07$ & +0.03 & $-1.48 \pm 0.05$ & +0.02 & $-1.03 \pm 0.16$ \\
\hline Mg II & 2 & $-1.56 \pm 0.14$ & -0.07 & $-1.59 \pm 0.09$ & -0.09 & $-1.83 \pm 0.15$ \\
\hline Si II & 4 & $-1.10 \pm 0.15$ & +0.39 & $-1.20 \pm 0.12$ & +0.30 & $-1.31 \pm 0.24$ \\
\hline $\mathrm{Ca} \mathrm{I}$ & 2 & $-0.80 \pm 0.35$ & +0.69 & $-0.93 \pm 0.25$ & +0.57 & $-0.09 \pm 0.33$ \\
\hline $\mathrm{Ca}$ II & 1 & $-0.49 \pm 0.10$ & +1.00 & $-0.62 \pm 0.10$ & +0.88 & $-0.32 \pm 0.10$ \\
\hline Sc II & 7 & $-0.99 \pm 0.25$ & +0.50 & $-1.04 \pm 0.25$ & +0.45 & $-0.73 \pm 0.25$ \\
\hline Ti II & 51 & $-0.75 \pm 0.18$ & +0.74 & $-0.83 \pm 0.25$ & +0.67 & $-0.59 \pm 0.19$ \\
\hline $\mathrm{Cr}$ II & 8 & $-1.41 \pm 0.12$ & +0.08 & $-1.47 \pm 0.12$ & +0.03 & $-1.38 \pm 0.12$ \\
\hline $\mathrm{Fe} \mathrm{I}$ & 13 & $-1.48 \pm 0.15$ & +0.02 & $-1.53 \pm 0.26$ & +0.02 & $-0.99 \pm 0.14$ \\
\hline $\mathrm{Fe}$ II & 28 & $-1.50 \pm 0.21$ & +0.00 & $-1.46 \pm 0.22$ & +0.02 & $-1.52 \pm 0.22$ \\
\hline Sr II & 1 & $-2.19 \pm 0.10$ & -0.70 & $-2.21 \pm 0.10$ & -0.71 & $-1.65 \pm 0.12$ \\
\hline Y II & 2 & $-1.20 \pm 0.17$ & +0.29 & $-1.30 \pm 0.12$ & +0.37 & $-0.64 \pm 0.14$ \\
\hline $\mathrm{Ba}$ II & 2 & $-1.21 \pm 0.05$ & +0.28 & $-1.23 \pm 0.04$ & +0.26 & $-0.61 \pm 0.10$ \\
\hline
\end{tabular}

\subsection{Balmer lines}

We tried to estimate the $T_{\text {eff }}$ and $\log g$ from the analysis of Balmer lines in the spectrum of ZNG 4 using the Kurucz spectral atlas for Balmer lines (Kurucz CDROM 13, 1993).

We could not get a satisfactory fit between the observed and theoretical Balmer line profiles with the atmospheric parameters $T_{\text {eff }}=8500 \mathrm{~K}, \log g=2.5, V_{\mathrm{t}}=2.5 \mathrm{~km} \mathrm{~s}^{-1}$ and $[\mathrm{Fe} / \mathrm{H}]=-1.5$. We also tried models that take into account the alpha element enhancements and no convective overshooting (ANOVER models: http://kurucz.harvard.edu/ grids/gridm15ANOVER/bm15ak2nover . dat). They did not make any difference to the abovementioned atmospheric parameters (Fig. 4). The best fit to $\mathrm{H}_{\beta}$ profile was obtained with 


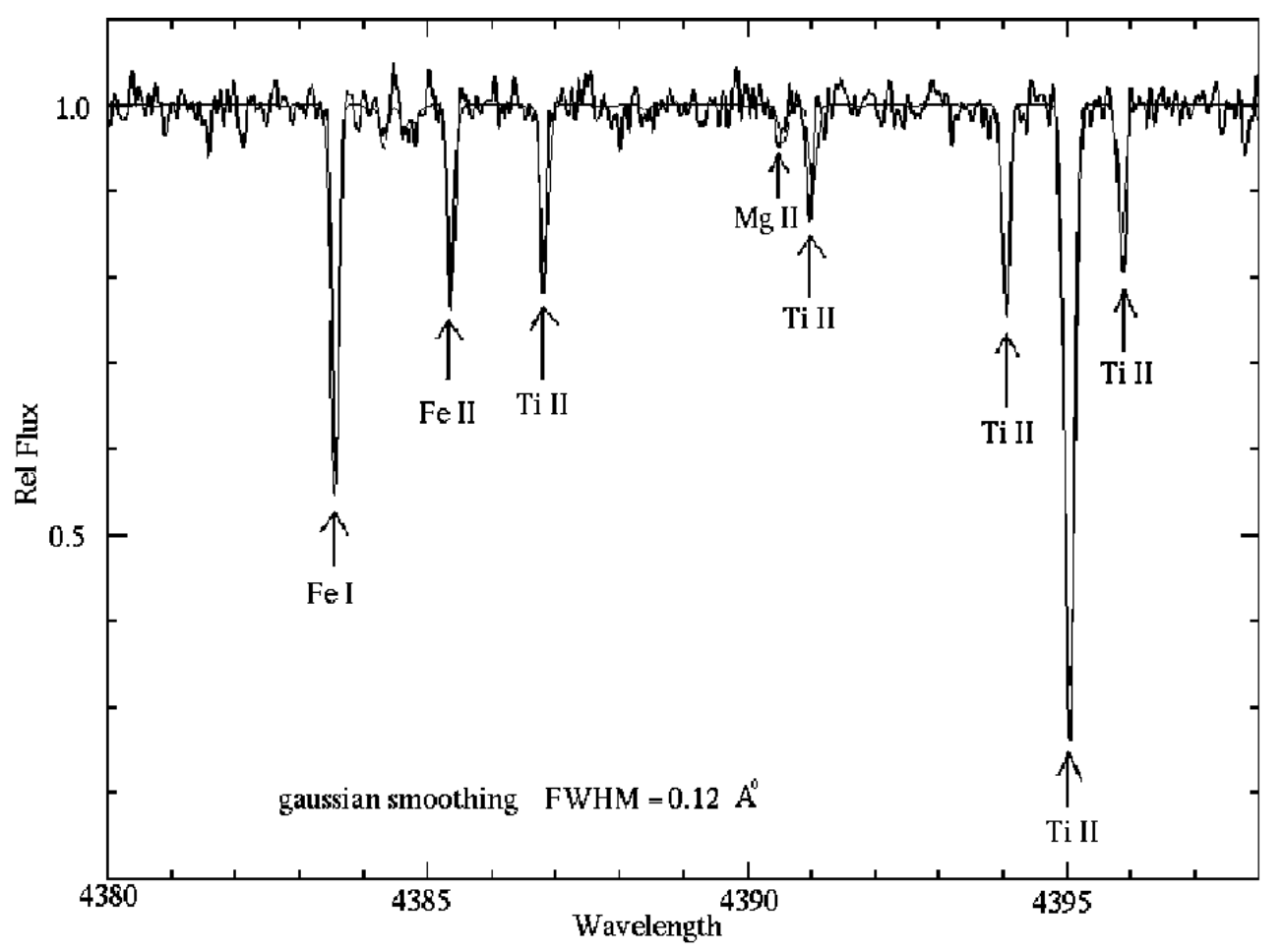

Fig. 3. Synthetic spectrum calculated with the atmospheric parameters $\left(T_{\text {eff }}=8500 \mathrm{~K}, \log g=2.5, V_{\mathrm{t}}=2.5 \mathrm{~km} \mathrm{~s}^{-1}\right)$ and abundances $($ Tables 1 and 3) is overplotted on the observed spectrum in the $4380 \AA-4400 \AA$ region.

the parameters $T_{\text {eff }}=8750 \mathrm{~K}, \log g=2.0, V_{\mathrm{t}}=2.0 \mathrm{~km} \mathrm{~s}^{-1}$ and $[\mathrm{Fe} / \mathrm{H}]=-1.5$ (Fig. 4). However, the excitation balance and ionization equilibrium for $\mathrm{Mg}$ and $\mathrm{Fe}$ lines could not be achieved with the above parameters (see the last column in Table 1) and the abundances of Mg I, Mg II and Fe I, Fe II were found to differ significantly (Table 1). Therefore, we chose the model atmosphere determined from the analysis of metal lines (i.e. $T_{\text {eff }}=8500 \mathrm{~K}, \log g=2.5, V_{\mathrm{t}}=2.5 \mathrm{~km} \mathrm{~s}^{-1}$ and $[\mathrm{Fe} / \mathrm{H}]=-1.5)$ to represent the atmosphere of the star.

The problem of fitting Balmer line profiles of HB stars has been mentioned by Grundahl et al. (1999) (and references therein). For stars being more luminous than HB stars, mass loss and/or extended atmosphere may influence the Balmer line profiles (Vink \& Cassisi 2002).

\section{Results}

The mean abundances of ZNG 4 relative to the Sun (Anders \& Grevesse 1989) are given in Table 1, together with the number of lines used in the analysis and the standard deviation of abundances estimated from individual species.

Analysis of $\mathrm{Mg}$ lines gives $[\mathrm{Mg} / \mathrm{H}]=-1.5$ which is the same as the M 13 cluster metallicity. (The equivalent widths of the Mg II lines at $4481 \AA$ (Table 3) were obtained by multiple gaussian fit to the lines in the observed spectrum). Silicon is overabundant compared to iron $([\mathrm{Si} / \mathrm{Fe}]=+0.4)$. Calcium and titanium are found to be overabundant $([\mathrm{Ca} / \mathrm{Fe}]=+0.8$ and $[\mathrm{Ti} / \mathrm{Fe}]=+0.75)$. There is a 0.5 dex difference in the abundances derived from the $\mathrm{Ca} I$ lines at $4226.73 \AA$ and $4454.78 \AA$ (Table 3). However, the abundance of $\mathrm{Ca}$ derived from the Ca II line at $5019.97 \AA$ is in agreement with that derived from the $\mathrm{Ca}$ I $4454.78 \AA$ line. The reason for the deviation in the abundance derived from the Ca I $4226.73 \AA$ line is not clear. It may be due to the relatively low signal to noise ratio of the data around this wavelength range. There seems to be no interstellar contribution to the Ca II line. Since the star has a radial velocity of $-257 \mathrm{~km} \mathrm{~s}^{-1}$, the stellar lines are well separated from the lines of interstellar origin. The abundance of $\mathrm{Cr}$ and $\mathrm{Fe}([\mathrm{Fe} / \mathrm{H}]=-1.48$ and $[\mathrm{Cr} / \mathrm{Fe}]=+0.09)$ are found to be close to the metallicity of the cluster. On the other hand, Sc is found to be overabundant $([\mathrm{Sc} / \mathrm{Fe}]=0.51)$. Na lines show an overabundance of 0.2 dex. We have detected one line of Sr II, two lines of Y II and two lines of Ba II. Sr seems to be underabundant $([\mathrm{Sr} / \mathrm{Fe}]=-0.70)$, while $\mathrm{Y}$ and $\mathrm{Ba}$ are overabundant $([\mathrm{Y} / \mathrm{Fe}]=+0.29$ and $[\mathrm{Ba} / \mathrm{Fe}]=+0.28)$.

We have detected the He I line at $4471.47 \AA$, which yields an abundance of $\log \epsilon(\mathrm{He})=10.44$ which implies an underabundance of 0.55 dex compared to the solar value. This is in agreement with the underabundane of He found in hot BHB stars (Moehler 1999; Moehler et al. 2003).

We have not detected $\mathrm{C}, \mathrm{N}$ and $\mathrm{O}$ lines in our spectrum of ZNG 4. Assuming an equivalent width of $5 \mathrm{~m} \AA$ as the detectable limit in our spectrum of ZNG 4, we find the upper limit of $[\mathrm{C} / \mathrm{Fe}]$ to be +0.32 dex (based on the C I $5052.17 \AA$ line), that of $[\mathrm{N} / \mathrm{Fe}]$ to be $+1.15 \mathrm{dex}$ (based on the N I $4214.80 \AA$ line) and that of $[\mathrm{O} / \mathrm{Fe}]$ to be $+0.01 \mathrm{dex}$ (based on the O I $6156.78 \AA$ line). Globular cluster stars show anticorrelation of sodium and oxygen abundances (Kraft et al. 1997). In ZNG 4 we find enhancement of sodium, therefore we expect an underabundance of oxygen. Also star to star abundance variations in the light elements $\mathrm{C}, \mathrm{N}, \mathrm{O}, \mathrm{Na}, \mathrm{Mg}$ and $\mathrm{Al}$ occur among the bright giants of a number of globular clusters (Ivans et al. 1999). The absence of $\mathrm{C}, \mathrm{N}$ and $\mathrm{O}$ lines in our spectrum of 


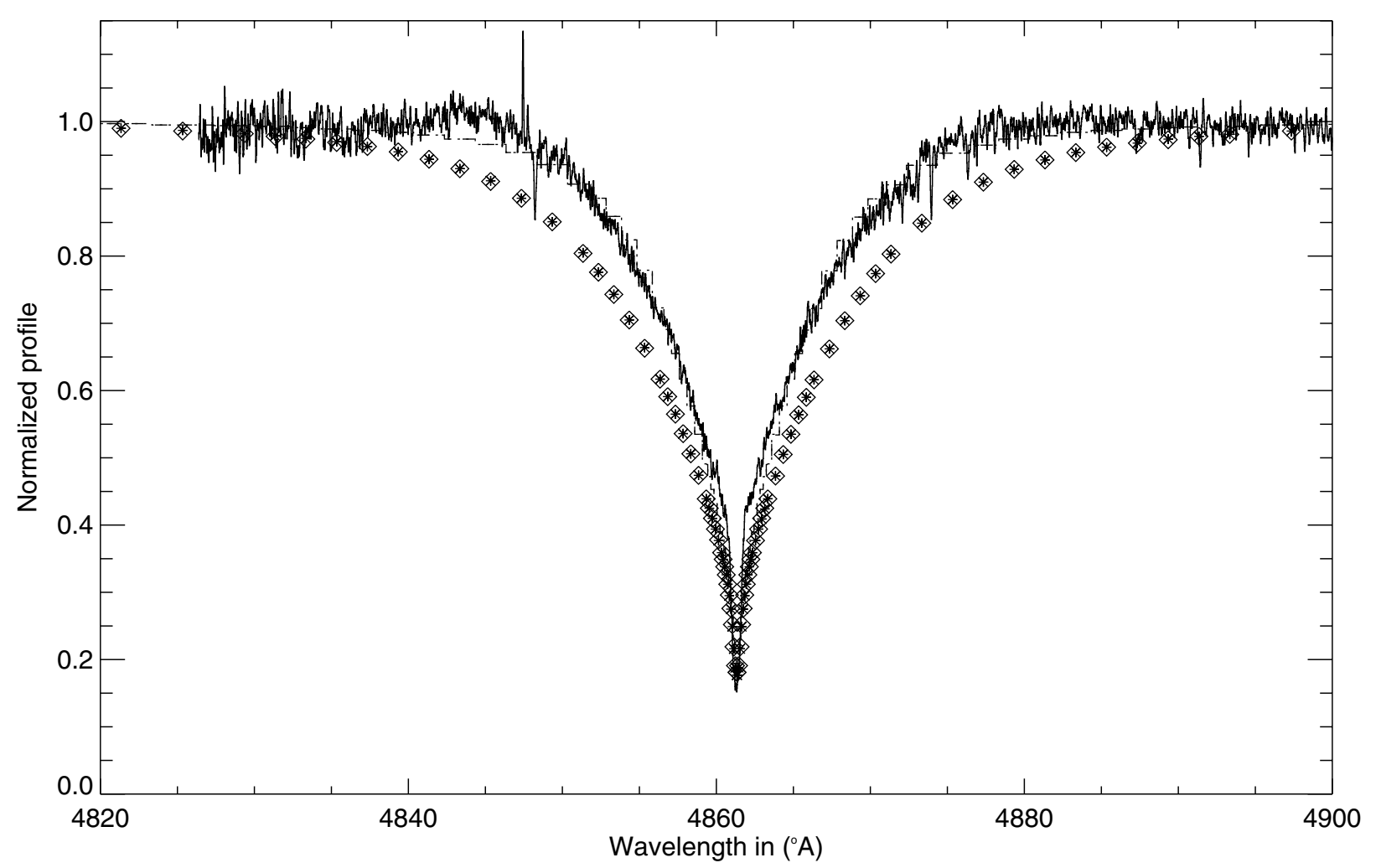

Fig. 4. Observed $\mathrm{H}_{\beta}$ profile compared with theoretical $\mathrm{H}_{\beta}$ profiles for 2 different model atmospheric parameters. Dots and dashes represent the model with $T_{\text {eff }}=8750 \mathrm{~K}, \log g=2.0,[\mathrm{M} / \mathrm{H}]=-1.5$ and $V_{\mathrm{t}}=2.0 \mathrm{~km} \mathrm{~s}^{-1}$ which fit the profile best. Open diamonds and asterixs represent the model $T_{\text {eff }}=8500 \mathrm{~K}, \log g=2.5,[\mathrm{M} / \mathrm{H}]=-1.5$ and $V_{\mathrm{t}}=2.5 \mathrm{~km} \mathrm{~s}^{-1}$, which does not fit the observed profile. No noticable differences were observed in the theoretical $\mathrm{H}_{\beta}$ profiles by considering ANOVER models for the same atmospheric parameters and are shown by dashes for the first set of parameters and asterix in the case of second set of parameters.

ZNG 4 may be due to the underabundance of these elements. A much higher resolution and high signal to noise ratio spectrum of $\mathrm{ZNG} 4$ may reveal the lines of $\mathrm{C}, \mathrm{N}$ and $\mathrm{O}$ lines if present.

\section{Discussion and conclusions}

The chemical composition of ZNG 4 shows significant deviations in element abundances from the expected metallicity of $\mathrm{M} 13$. Ti, $\mathrm{Ca}, \mathrm{Sc}$ and $\mathrm{Ba}$ are found to be relatively overabundant (Fig. 5) compared to RGB stars and cool BHB stars of M 13, whereas the abundances of $\mathrm{Mg}, \mathrm{Cr}$ and $\mathrm{Fe}$ are in agreement with the cluster metallicity.

From a study of 22 M 13 G-K giants, Kraft et al. $(1993,1997)$ found the abundances of $\mathrm{Fe}, \mathrm{Sc}, \mathrm{V}$ and $\mathrm{Ni}$ to be $[\mathrm{Fe} / \mathrm{H}]=-1.49,[\mathrm{Sc} / \mathrm{Fe}]=-0.10,[\mathrm{~V} / \mathrm{Fe}]=0.00$ and $[\mathrm{Ni} / \mathrm{Fe}]=-0.04$. They found $\mathrm{Ca}$ and $\mathrm{Ti}$ to be mildly overabundant $([\mathrm{Ca} / \mathrm{Fe}]=+0.24$ and $[\mathrm{Ti} / \mathrm{Fe}]=+0.29)$. Si was overabundant by +0.34 dex. Study of M 13 giants by Armosky et al. (1994) yields the average abundance of $\mathrm{Fe}, \mathrm{Y}$ and $\mathrm{Ba}:[\mathrm{Fe} / \mathrm{H}]=-1.49,[\mathrm{Y} / \mathrm{Fe}]=-0.12$ and $[\mathrm{Ba} / \mathrm{Fe}]=-0.04$, whereas in ZNG 4 we find significant overabundance of $\mathrm{Ca}$, $\mathrm{Sc}, \mathrm{Ti}, \mathrm{Y}$ and Ba compared to that found in M 13 giants. Also, overabundance of $\mathrm{Na}[+0.2 \mathrm{dex}]$ and absence of $\mathrm{O}$ lines support the anticorrelation of $\mathrm{Na}$ and $\mathrm{O}$ abundances found in $\mathrm{M} 13$ giants (Kraft et al. 1997).

Behr et al. (1999) have studied the BHB stars in M 13 on either side of the HB gap. They find the photospheric compositions and stellar rotation rates to vary strongly as a function of $T_{\text {eff }}$ of the stars. Among the cooler stars in their sample, at $T_{\text {eff }}$ of $8500 \mathrm{~K}$, the metal abundances are in rough agreement with the canonical cluster metallicity and the hotter stars with $T_{\text {eff }}$ greater than $10000 \mathrm{~K}$ show a deficiency of $\mathrm{He}$ and enhancement of $\mathrm{Fe}, \mathrm{Ti}, \mathrm{Cr}$ by a factor of 300 . However, $\mathrm{Mg}$ remains at the canonical cluster metallicity. In ZNG 4 also, $\mathrm{Mg}$ abundance is in agreement with the M 13 metallicity. Abundances similar to that found in BHB stars of M 13 were also found in BHB stars of M 15 (Behr et al. 2000b) and BHB stars of NGC 6752 (Glaspy et al. 1989; Moehler et al. 1999). The abundance anomalies in these BHB stars are most likely due to diffusion - the gravitational settling of helium (Greestein et al. 1967) and radiative levitation of metal atoms (Michaud et al. 1983). Rotational velocities ( $v \sin i$ ) appear to have a bimodal distribution in cooler BHB stars, whereas the hotter BHB stars with $T_{\text {eff }}$ greater than $10000 \mathrm{~K}$ are found to be slow rotators.

However, the abundances and rotational velocity of ZNG 4 are contrary to what is expected for stars on the red side of the HB gap at $11000 \mathrm{~K}$. It is a slow rotator $\left(v \sin i=7 \mathrm{~km} \mathrm{~s}^{-1}\right)$. It shows underabundance of $\mathrm{He}$ and overabundances of $\mathrm{Ti}, \mathrm{Ca}$, Sc and Ba. In Table 2 and Fig. 5, we have compared the abundances of ZNG 4 with the abundances of M 13 BHB stars of $T_{\text {eff }} 7681 \mathrm{~K}$ (J 11) and $T_{\text {eff }} 12750 \mathrm{~K}$ (WF4-3485) (Behr 2000c) and with the abundances of the RGB star L262 (Cavallo \& Nagar 2000). In Table 2, the last column shows the mean 
Table 2. Comparison of the abundances of ZNG 4 with the abundances of M 13 BHB and RGB stars.

\begin{tabular}{|c|c|c|c|c|c|}
\hline Element & $\begin{array}{c}\text { ZNG } 4 \\
{[\mathrm{Fe} / \mathrm{H}]=-1.49} \\
T_{\text {eff }}=8500 \mathrm{~K} \\
\log g=2.5\end{array}$ & $\begin{array}{c}\mathrm{M} 13 / \mathrm{J} 11(\mathrm{BHB})^{*} \\
{[\mathrm{Fe} / \mathrm{H}]=-1.82} \\
T_{\text {eff }}=7681 \mathrm{~K} \\
\log g=3.1\end{array}$ & $\begin{array}{c}\mathrm{M} 13 / \mathrm{WF} 4-3485(\mathrm{BHB})^{*} \\
{[\mathrm{Fe} / \mathrm{H}]=+0.02} \\
T_{\text {eff }}=12750 \mathrm{~K} \\
\log g=4.1\end{array}$ & $\begin{array}{c}\text { M 13/ L262 (RGB) } \\
{[\mathrm{Fe} / \mathrm{H}]=-1.61} \\
T_{\text {eff }}=4160 \mathrm{~K} \\
\log g=0.50\end{array}$ & $\begin{array}{c}\text { Mean } \\
\text { RGB abundances* } \\
\text { in M } 13\end{array}$ \\
\hline & {$[\mathrm{X} / \mathrm{H}]$} & {$[\mathrm{X} / \mathrm{H}]$} & {$[\mathrm{X} / \mathrm{H}]$} & {$[\mathrm{X} / \mathrm{H}]$} & {$[\mathrm{X} / \mathrm{H}]$} \\
\hline $\mathrm{He}$ & -0.55 & $<-0.26$ & $-1.49 \pm 0.17$ & $\ldots$ & $\ldots$ \\
\hline $\mathrm{Na}$ & -1.28 & $\ldots$ & $\ldots$ & $-1.27 \pm 0.11$ & $-1.37 \pm 0.04$ \\
\hline $\mathrm{Mg}$ & -1.51 & $-1.50 \pm 0.16$ & $-1.62 \pm 0.14$ & $-1.51 \pm 0.14$ & $-1.46 \pm 0.03$ \\
\hline $\mathrm{Si}$ & -1.10 & $<-1.23$ & $-1.43 \pm 0.07$ & $-1.16 \pm 0.14$ & $-1.30 \pm 0.02$ \\
\hline $\mathrm{Ca}$ & -0.64 & $-1.72 \pm 0.11$ & $-1.66 \pm 0.15$ & $-1.39 \pm 0.14$ & $-1.34 \pm 0.01$ \\
\hline $\mathrm{Sc}$ & -0.99 & $-1.63 \pm 0.10$ & $<+1.10$ & $\ldots$ & $-1.67 \pm 0.01$ \\
\hline $\mathrm{Ti}$ & -0.75 & $-1.32 \pm 0.06$ & $-0.53 \pm 0.16$ & $-1.30 \pm 0.20$ & $-1.32 \pm 0.02$ \\
\hline $\mathrm{Cr}$ & -1.41 & $-1.71 \pm 0.12$ & $<-0.12$ & $\ldots$ & $\ldots$ \\
\hline $\mathrm{Fe}$ & -1.49 & $-1.82 \pm 0.10$ & $+0.02 \pm 0.20$ & $-1.61 \pm 0.10$ & $-1.60 \pm 0.01$ \\
\hline $\mathrm{Sr}$ & -2.19 & $-2.03 \pm 0.10$ & $<+1.75$ & $\ldots$ & $\ldots$ \\
\hline $\mathrm{Y}$ & -1.20 & $<-0.97$ & $<+2.92$ & $\ldots$ & $-1.61 \pm 0.04$ \\
\hline $\mathrm{Ba}$ & -1.21 & $-1.68 \pm 0.10$ & $<+3.26$ & $\ldots$ & $-1.53 \pm 0.05$ \\
\hline
\end{tabular}

* Abundances of M 13 BHB stars are from Behr (2000c) and abundances of M 13 RGB star are from Cavallo \& Nagar (2000). In the last column, the mean abundances of elements from Na to Fe in M 13 RGB stars are from Kraft et al. (1997) and that of Y and Ba are from Armosky et al. (1994).

RGB abundances in M 13, where elements from $\mathrm{Na}$ to $\mathrm{Fe}$ are taken from Kraft et al. (1997) and Y and Ba abundances are from Armosky et al. (1994). It is evident from the abundances listed in Table 2 and from Fig. 5 that ZNG 4 shows overabundance of metals compared to that of a M 13 RGB star and also when compared to that of a M 13 BHB star of similar temperature. These results indicate that in $\mathrm{ZNG} 4$, diffusion and radiative levitation of elements may be in operation. Slowly rotating HB stars are also seen on the cooler side of HB gap, but abundance anomalies start from $11000 \mathrm{~K}$ (Moehler et al. 1999; Behr et al. 2000b). This implies that ZNG 4 may have the properties of the stars on the blue side of the HB gap although it has $T_{\text {eff }}$ of $8500 \mathrm{~K}$. In this regard, more accurate determination of abundances of these elements in ZNG 4 and similar stars in M 13 is needed to confirm our results and conclusions.

This may be explained in two ways. One is that, for some stars in M 13, the onset of diffusion seems to start at lower $T_{\text {eff }}(\approx 8500 \mathrm{~K})$. The other argument would be that the star has evolved from the blue side of the HB gap and is moving towards the red with higher luminosity as indicated by the postHB evolutionary tracks of Gingold (1976) and Dorman et al. (1993).

The BHB stars hotter than $11500 \mathrm{~K}$ typically show strong photospheric helium depletions due to gravitational settling (Moehler et al. 2000, 2003). The calculations of Michaud et al. (1983) indicate that helium depletion should be accompanied by photospheric enhancement of metals, since the same stable atmosphere that permits gravitational settling also permits the levitation of elements with large radiative cross sections. The depletion of helium and overabudance of some of the metals in the photosphere of $\mathrm{ZNG} 4$ is in qualitative agreement with the calculations of Michaud et al. (1983).
Recently, Turcotte et al. (1998) and Richer et al. (2000) made diffusion simulations to explain the abundance patterns of chemically peculiar A and F stars. Their predicted abundance patterns are qualitatively similar to that found in $\mathrm{ZNG} 4$. However, none of the recent diffusion studies treated the cases of BHB stars and post-HB stars. This phenomenon may be related to the disappearance of surface convection and hence to the formation of a stable stellar atmospheres. HB stars and postHB stars cooler than $T_{\text {eff }}=6300 \mathrm{~K}$ have deep convective envelopes (Sweigart 2002). Hotter than this temperature the envelope convection breaks into distinct shells associated with the ionization of $\mathrm{H}$ and $\mathrm{He}$. Note that the surface convection disappears at $11000 \mathrm{~K}$ (Sweigart 2002) and BHB stars hotter than this show moderate to severe abundance anomalies.

ZNG 4 has a $V$ magnitude of 13.964 (Paltrinieri et al. 1998). Considering the distance modulus of M 13 to be 14.42 and $E(B-V)$ towards M 13 to be 0.02 (Kraft \& Ivans 2003), we estimated the absolute magnitude $\left(M_{v}\right)$ of the star to be -0.522 . For stars with $T_{\text {eff }}$ around $8500 \mathrm{~K}$, the bolometric correction (BC) is negligible (Flower 1996). Considering $B C=0$, we get the bolometric magnitude $\left(M_{\mathrm{bol}}\right)$ to be -0.522 , which corresponds to a luminosity of $127 L_{\odot}\left[\log \frac{L}{L_{\odot}}=2.18\right]$. Using the equation connecting the mass, effective temperature and bolometric magnitude, we find the surface gravity, $\log g=2.6$ (assuming the mass of ZNG 4 to be $0.5 M_{\odot}$ ), which agrees well with the value estimated from the analysis of the spectrum of ZNG 4 .

The post-AGB star Barnard $29\left[\log \frac{L}{L_{0}}=3.3\right]$ which is a member of M 13 is more luminous than $\mathrm{ZNG} 4$. The abundance pattern of ZNG 4 is very different from that of the post-AGB star Barnard 29 (Conlon et al. 1994; Moehler et al. 1998). The M 13 BHB stars with $T_{\text {eff }}$ around $8500 \mathrm{~K}$ have a luminosity 


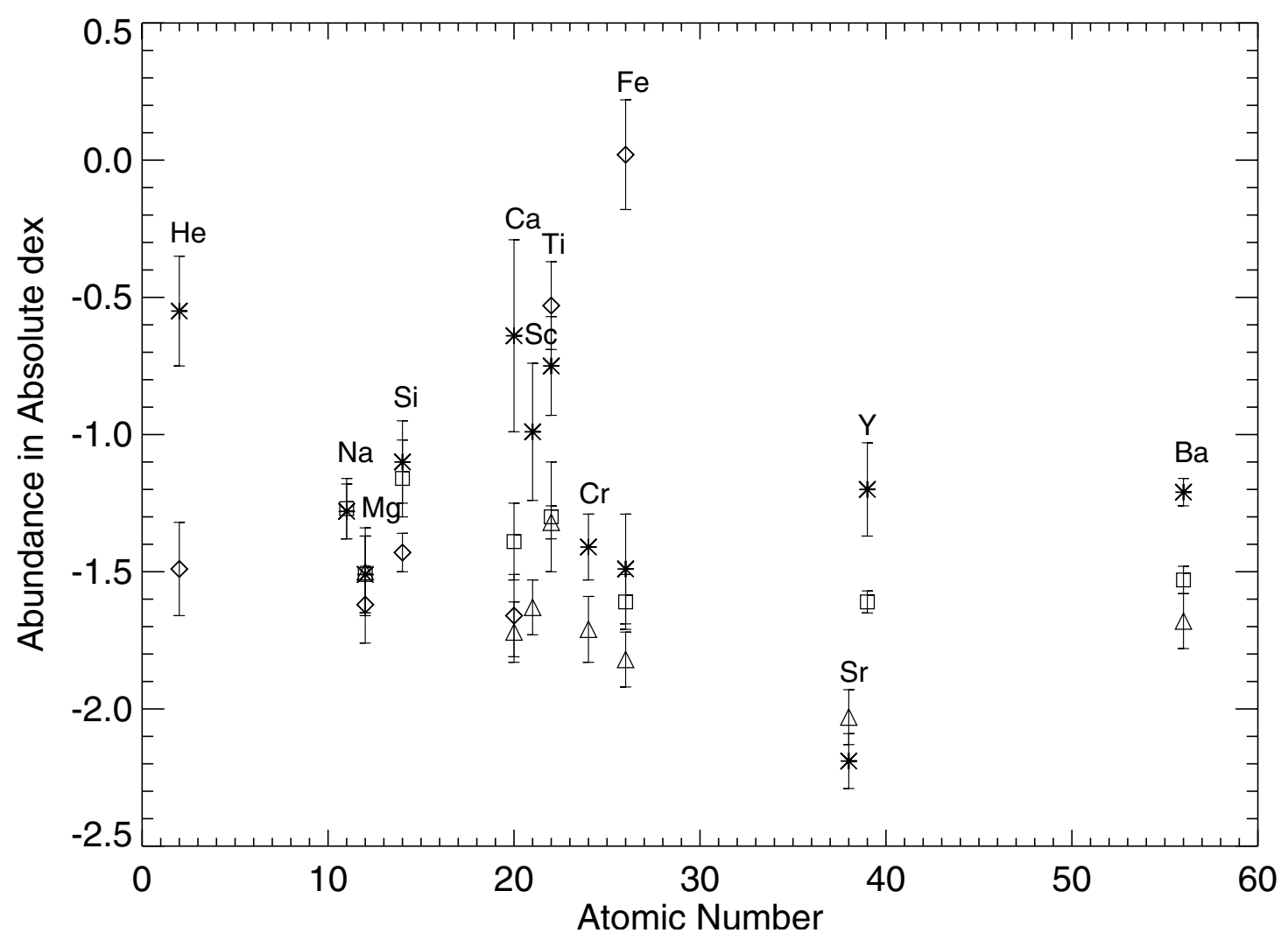

Fig. 5. The abundances of different elements in ZNG 4 (represented by asterix) compared with M 13/ J11 (BHB star; triangles), M 13/WF4-3485 (hot BHB star; diamonds) and that of M 13/ L262 (RGB star; squares) with their error bars (Table 2). In the cases where only upper limit of the abundances are given (3rd and 4th column of Table 2), those elements are not shown in the above figure. Average abundances of $\mathrm{Y}$ and Ba in M 13 RGB stars are from Armosky et al. (1994).

of about $40 L_{\odot}$, whereas ZNG 4 is more luminous by about a factor of 3, which indicates that ZNG 4 can be classified as a supra horizontal branch star (post-HB). Stars that lie 1.5 magnitude above the HB stars are classified as supra horizontal branch (SHB) stars in the photometric studies of M 13 (Zinn 1974) and NGC 6522 (Shara et al. 1998). No detailed abundance analysis of SHB stars in globular clusters is available to compare with the abundances of ZNG 4.

Since ZNG 4 is a post-HB star and it has evolved from a hot BHB star stage and may had severe abundance anomalies similar to those found in the hot BHB stars of M 13 (Table 2). The present $T_{\text {eff }}=8500 \mathrm{~K}$ of ZNG 4 indicates that thin layers of subsurface convection if present may have diluted the severe abundance anomalies due to diffusion and radiative levitation that took place during its hot BHB stage of evolution. It is important to derive the chemical composition of a significant sample of post-HB stars hotter than $11000 \mathrm{~K}$ and much cooler than $11000 \mathrm{~K}$ to further understand the role of diffusion, radiative levitation, rotation and convection during the post-HB stage of evolution.

Acknowledgements. We would like to thank Dr. B.Dorman and Dr. B. Behr for helpful information on M 13 BHB stars, Dr. F. R. Ferraro for kindly providing the table of $B$ and $V$ magnitudes of M 13 cluster stars and the referee Dr. S. Moehler for helpful comments.

\section{References}

Anders, E., \& Grevesse, N. 1989, Geochim. Cosmochim. Acta, 53, 197

Armosky, B. J., Sneden, C., Langer, G. E., \& Kraft, R. P. 1994, AJ, 108,1364

Behr, B. B., Cohen, J. G., McCarthy, J. K., \& Djorgovski, S. G. 1999, ApJ, 517L, 135

Behr, B. B., Djorgovski, S. G., Cohen, J. G., et al. 2000a, ApJ, 528, 849

Behr, B. B., Cohen, J. G., \& McCarthy, J. K. 2000b, ApJ, 531, L37

Behr, B. B. 2000c, Ph.D. Thesis, California Institute of Technology

Brocato, E., Matteucci, F., Mazzitelli, I., \& Tornambe, A. 1990, ApJ, 349,458

Cavallo, R. M., \& Nagar, N. M. 2000, AJ, 120, 1364

Conlon, E. S., Dufton, P. L., \& Keenan, F. P. 1994, A\&A, 290, 897

de Boer, K. S. 1985, A\&A, 142, 321

de Boer, K. S. 1987, The second conference on faint blue stars (L. Davis Press), IAU Colloq., 95, 95

Dorman, B., Rood, R. T., \& O’Connell, R. W. 1993, ApJ, 419, 596

Flower, P. J. 1996, ApJ, 469, 355

Ferraro, F. R., Paltrinieri, B., Fuci Pecci, F., Dorman, B., \& Rood, R. T. 1997, ApJ, 500, 311

Gingold, R. A. 1976, ApJ, 204, 116

Glaspey, J. W., Michaud, G., Moffat, A. F. J., \& Demers, S. 1989, ApJ, 339,926

Greenstein, G. S., Truran, J. W., \& Cameron, A. G. W. 1967, Nature, 213,871

Grundahl, F., Catelan, M., Landsman, W. B., et al. 1999, ApJ, 524, 242 
Gonzalez, G., \& Wallerstein, G. 1994, AJ, 108, 1325

Harris, H. C., Nemec, J. M., \& Hesser, J. E. 1983, PASP, 95, 256

Ivans, I. I., Sneden, C., Kraft, R. P., et al. 1999, ApJ, 118, 1273

Kraft, R. P., Sneden, C., Langer, G. E., \& Shetrone, M. D. 1993, AJ, 106,1490

Kraft, R. P., Sneden, C., Smith, G. H., et al. 1997, AJ, 113, 279

Kraft, R. P., \& Ivans, I. I. 2003, PASP, 115, 143

Kurucz, R. L. 1993, Kurucz CD-ROMS (Cambridge: Smithsonian Astophys. Obs.)

Michaud, G., Vauclair, G., \& Vauclair, S. 1983, ApJ, 267, 256

Moehler, S., Heber, M., Lemke, M., \& Napiwotzki, R. 1998, A\&A, 339, 537

Moehler, S. 1999, Rev. Modern Astron., 12, 281

Moehler, S., Sweigart, A. V., Landsman, W. B., Heber, U., \& Catelan, M. 1999, A\&A, 346, L1

Moehler, S., Sweigart, A. V., Landsman, W. B., \& Heber, U. 2000, A\&A, 360, 120

Moehler, S., Landsman, W. B., Sweigart, A. V., \& Grundahl, F. 2003, A\&A, 405, 135
Moore, C. E. 1945, A Multiplet Table of Astrophysical Interest, Part I, Table of Multiplets, revised ed. (Princeton: Princeton Univ. Obs.) Noguchi, K., Aoki, W., Kawanomoto, S., et al. 2002, PASJ, 54, 855

Paltrinieri, B., Ferraro, F. R., Carretta, E., \& Fusi Pecci, F. 1998, MNRAS, 293, 434

Peterson, R. C. 1983, ApJ, 275, 737

Peterson, R. C., Rood, R. T., \& Crocker, D. A. 1995, ApJ, 453, 21

Preston, G. W., \& Sneden, C. 2000, AJ, 120, 1014

Rey, S.-C., Yoon, S.-J., Lee, Y.-W., Chaboyer, B., \& Sarajedini, A. 2001, AJ, 122, 3219

Richer, J., Michaud, G., \& Turcotte, S. 2000, ApJ, 529, 338

Shara, M. M., Drissen, L., Rich, R. M., et al. 1998, ApJ, 495, 796

Sneden, C. 1973, ApJ, 184, 839

Sweigart, A. V., Mengel, J. G., \& Demarque, P. 1974, A\&A, 30, 13

Sweigart, A. V. 2002, IAU Highlights of Astronomy, 12, 293

Turcotte, S., Richer, J., \& Michaud, G. 1998, ApJ, 504, 559

Vink, J. S., \& Cassisi, S. 2002, A\&A, 392, 553

Zinn, R. J., Newell, E. B., \& Gibson, J. B. 1972, A\&A, 18, 390

Zinn, R. J. 1974, ApJ, 193, 593 
S. Ambika et al.: Chemical composition of UV-bright star ZNG 4 in M 13, Online Material p 1

\section{Online Material}


Table 3. Data for spectral lines measured in the spectrum of ZNG 4 Table 3. continued. in $\mathrm{M} 13$.

\begin{tabular}{|c|c|c|c|c|}
\hline$\lambda_{\text {lab }}($ in $\AA)$ & LEP $(\mathrm{eV})$ & $\log g f$ & $E W(\mathrm{~m} \AA)$ & $\log \epsilon$ \\
\hline \multicolumn{5}{|l|}{$\mathrm{He} \mathrm{I}$} \\
\hline 4471.47 & 20.96 & -0.278 & 11.3 & 10.44 \\
\hline \multicolumn{5}{|l|}{$\mathrm{Na} \mathrm{I}$} \\
\hline 5889.95 & 0.00 & 0.117 & 63.9 & 5.05 \\
\hline 5895.92 & 0.00 & -0.184 & 40.6 & 5.04 \\
\hline \multicolumn{5}{|l|}{$\mathrm{Mg} \mathrm{I}$} \\
\hline 5167.32 & 2.71 & -1.030 & 31.9 & 6.19 \\
\hline 5172.68 & 2.71 & -0.402 & 70.2 & 6.09 \\
\hline 5183.60 & 2.72 & -0.180 & 85.8 & 6.07 \\
\hline \multicolumn{5}{|l|}{$\mathrm{Mg}$ II } \\
\hline 4481.13 & 8.86 & 0.740 & 104.1 & 6.12 \\
\hline 4481.32 & 8.86 & 0.590 & 80.0 & 5.92 \\
\hline \multicolumn{5}{|l|}{ Si II } \\
\hline 5041.02 & 10.07 & 0.291 & 27.5 & 6.59 \\
\hline 5055.98 & 10.07 & 0.593 & 25.5 & 6.24 \\
\hline 6347.11 & 8.12 & 0.297 & 84.2 & 6.49 \\
\hline 6371.37 & 8.12 & -0.003 & 64.1 & 6.48 \\
\hline \multicolumn{5}{|l|}{$\mathrm{Ca}$ I } \\
\hline 4226.73 & 0.00 & 0.265 & 76.7 & 5.21 \\
\hline 4454.78 & 1.90 & 0.335 & 27.7 & 5.71 \\
\hline \multicolumn{5}{|l|}{$\mathrm{Ca}$ II } \\
\hline 5019.97 & 7.51 & -0.501 & 18.6 & 5.77 \\
\hline \multicolumn{5}{|l|}{ Sc II } \\
\hline 4246.82 & 0.32 & 0.242 & 54.5 & 1.58 \\
\hline 4314.08 & 0.62 & -0.096 & 47.8 & 2.03 \\
\hline 4320.73 & 0.60 & -0.252 & 49.7 & 2.21 \\
\hline 4325.00 & 0.59 & -0.442 & 35.9 & 2.18 \\
\hline 4374.46 & 0.62 & -0.418 & 39.8 & 2.23 \\
\hline 4400.39 & 0.60 & -0.536 & 32.3 & 2.21 \\
\hline 4415.56 & 0.59 & -0.668 & 31.5 & 2.32 \\
\hline \multicolumn{5}{|l|}{ Ti II } \\
\hline 4161.53 & 1.08 & -2.160 & 20.8 & 4.27 \\
\hline 4163.65 & 2.59 & -0.210 & 76.3 & 4.22 \\
\hline 4171.91 & 2.60 & -0.270 & 75.0 & 4.27 \\
\hline 4287.87 & 1.08 & -1.820 & 38.8 & 4.26 \\
\hline 4290.22 & 1.17 & -0.930 & 91.1 & 4.16 \\
\hline 4294.10 & 1.08 & -0.880 & 95.2 & 4.12 \\
\hline 4300.05 & 1.18 & -0.490 & 129.9 & 4.43 \\
\hline 4301.91 & 1.16 & -1.200 & 78.7 & 4.25 \\
\hline 4307.86 & 1.17 & -1.100 & 62.6 & 3.93 \\
\hline 4312.86 & 1.18 & -1.090 & 77.5 & 4.13 \\
\hline 4314.98 & 1.16 & -1.120 & 69.8 & 4.04 \\
\hline 4320.96 & 1.17 & -1.900 & 43.4 & 4.47 \\
\hline 4330.24 & 2.05 & -1.800 & 21.6 & 4.57 \\
\hline 4330.69 & 1.18 & -2.060 & 24.3 & 4.30 \\
\hline 4344.29 & 1.08 & -1.930 & 19.8 & 3.99 \\
\hline 4350.83 & 2.06 & -1.810 & 12.6 & 4.32 \\
\hline 4367.66 & 2.59 & -0.870 & 34.9 & 4.28 \\
\hline 4386.84 & 2.60 & -0.940 & 27.2 & 4.21 \\
\hline 4391.03 & 1.23 & -2.240 & 19.2 & 4.38 \\
\hline 4394.05 & 1.22 & -1.770 & 37.7 & 4.28 \\
\hline 4395.03 & 1.08 & -0.510 & 142.4 & 4.62 \\
\hline 4395.85 & 1.24 & -1.970 & 31.7 & 4.39 \\
\hline 4399.77 & 1.24 & -1.220 & 78.3 & 4.31 \\
\hline 4407.68 & 1.22 & -2.430 & 10.4 & 4.27 \\
\hline 4411.07 & 3.10 & -0.670 & 23.0 & 4.18 \\
\hline 4417.72 & 1.17 & -1.230 & 76.0 & 4.23 \\
\hline 4418.33 & 1.24 & -1.990 & 25.0 & 4.27 \\
\hline
\end{tabular}

\begin{tabular}{lllll}
\hline \hline$\lambda_{\text {lab }}$ (in $\AA$ ) & LEP $(\mathrm{eV})$ & $\log g f$ & $E W(\mathrm{~mA})$ & $\log \epsilon$ \\
\hline Ti II & & & & \\
4421.94 & 2.06 & -1.580 & 14.6 & 4.16 \\
4443.79 & 1.08 & -0.700 & 118.3 & 4.33 \\
4450.48 & 1.08 & -1.510 & 56.7 & 4.20 \\
4464.45 & 1.16 & -1.810 & 39.8 & 4.31 \\
4488.33 & 3.12 & -0.510 & 40.0 & 4.36 \\
4529.47 & 1.57 & -1.650 & 27.1 & 4.21 \\
4533.97 & 1.24 & -0.540 & 126.3 & 4.42 \\
4549.62 & 1.58 & -0.220 & 102.1 & 3.89 \\
4563.76 & 1.22 & -0.790 & 108.1 & 4.32 \\
4571.97 & 1.57 & -0.230 & 69.9 & 3.42 \\
4589.96 & 1.24 & -1.620 & 38.7 & 4.14 \\
4763.88 & 1.22 & -2.360 & 18.9 & 4.47 \\
4779.98 & 2.05 & -1.260 & 32.9 & 4.24 \\
4798.52 & 1.08 & -2.670 & 12.9 & 4.49 \\
4805.09 & 2.06 & -0.960 & 52.1 & 4.24 \\
4874.01 & 3.10 & -0.900 & 18.0 & 4.27 \\
4911.19 & 3.12 & -0.650 & 28.4 & 4.28 \\
5129.15 & 1.89 & -1.300 & 33.4 & 4.17 \\
5154.07 & 1.57 & -1.780 & 26.9 & 4.29 \\
5185.91 & 1.89 & -1.370 & 26.2 & 4.10 \\
5188.68 & 1.58 & -1.050 & 68.5 & 4.20 \\
5226.54 & 1.57 & -1.230 & 52.1 & 4.16 \\
5336.77 & 1.58 & -1.630 & 31.9 & 4.25 \\
5381.02 & 1.57 & -1.970 & 21.9 & 4.38 \\
Cr II & & & & \\
4554.99 & 4.07 & -1.282 & 14.8 & 4.40 \\
4558.65 & 4.07 & -0.449 & 44.5 & 4.18 \\
4588.20 & 4.07 & -0.627 & 34.6 & 4.20 \\
4616.63 & 4.07 & -1.361 & 15.0 & 4.48 \\
4618.80 & 4.07 & -0.840 & 26.1 & 4.25 \\
4634.07 & 4.07 & -0.990 & 19.1 & 4.23 \\
4824.13 & 3.87 & -0.970 & 20.6 & 4.11 \\
5237.33 & 4.07 & -1.160 & 13.0 & 4.21 \\
Fe I & & & & \\
4143.87 & 1.56 & -0.511 & 23.2 & 6.03 \\
4199.10 & 3.05 & 0.155 & 20.4 & 6.30 \\
4202.03 & 1.49 & -0.708 & 20.8 & 6.11 \\
4260.47 & 2.40 & 0.109 & 22.3 & 5.95 \\
4271.76 & 1.49 & -0.164 & 39.6 & 5.93 \\
4325.76 & 1.61 & 0.006 & 48.0 & 5.96 \\
4383.55 & 1.49 & 0.200 & 59.2 & 5.84 \\
4415.12 & 1.61 & -0.615 & 20.5 & 6.08 \\
4891.49 & 2.85 & -0.112 & 15.0 & 6.24 \\
4920.50 & 2.83 & 0.068 & 13.6 & 6.00 \\
4957.60 & 2.81 & 0.233 & 19.4 & 6.00 \\
5269.54 & 0.86 & -1.321 & 8.7 & 5.82 \\
5328.04 & 0.92 & -1.466 & 14.3 & 6.24 \\
Fe II & & & & \\
4173.46 & 2.58 & -2.740 & 62.2 & 6.42 \\
4178.86 & 2.58 & -2.500 & 43.0 & 5.90 \\
4233.17 & 2.58 & -1.900 & 90.5 & 5.99 \\
4351.77 & 2.70 & -3.010 & 16.5 & 5.95 \\
4416.89 & 2.70 & -2.020 & 54.1 & 5.66 \\
4489.18 & 2.78 & -2.680 & 31.0 & 6.01 \\
4491.40 & 2.83 & -2.410 & 41.3 & 5.91 \\
\hline & & -2.970 & 24.0 & 6.18 \\
& & & & 6.03 \\
\hline
\end{tabular}


Table 3. continued.

\begin{tabular}{lllll}
\hline \hline$\lambda_{\text {lab }}($ in $\AA)$ & LEP $(\mathrm{eV})$ & $\log g f$ & $E W(\mathrm{~mA})$ & $\log \epsilon$ \\
\hline Fe II & & & & \\
4508.29 & 2.86 & -2.250 & 43.9 & 5.84 \\
4515.34 & 2.84 & -2.450 & 34.4 & 5.87 \\
4520.22 & 2.81 & -2.600 & 33.0 & 5.98 \\
4522.63 & 2.84 & -2.030 & 43.0 & 5.60 \\
4549.47 & 2.83 & -2.020 & 80.0 & 6.10 \\
4555.89 & 2.83 & -2.160 & 46.2 & 5.76 \\
4576.34 & 2.84 & -2.920 & 18.9 & 6.01 \\
4582.84 & 2.84 & -3.090 & 16.1 & 6.10 \\
4583.84 & 2.81 & -1.860 & 79.8 & 5.92 \\
4629.34 & 2.81 & -2.330 & 45.9 & 5.91 \\
4923.93 & 2.89 & -1.320 & 121.3 & 6.14 \\
5018.44 & 2.89 & -1.220 & 127.4 & 6.15 \\
5169.03 & 2.89 & -1.303 & 134.4 & 6.37 \\
5197.58 & 3.23 & -2.100 & 45.9 & 5.96 \\
5234.62 & 3.22 & -2.230 & 47.6 & 6.11 \\
5276.00 & 3.20 & -1.940 & 57.3 & 5.95 \\
5316.61 & 3.15 & -1.850 & 67.0 & 5.97 \\
5362.87 & 3.20 & -2.739 & 29.6 & 6.31 \\
6456.38 & 3.90 & -2.100 & 45.8 & 6.46 \\
Sr II & & & & \\
4215.52 & 0.00 & -0.145 & 19.8 & 0.71 \\
Y II & & & & \\
4177.53 & 0.41 & -0.160 & 8.98 & 1.09 \\
4374.94 & 0.41 & 0.160 & 11.10 & 0.86 \\
Ba II & & & & \\
4554.03 & 0.00 & 0.170 & 17.03 & 0.95 \\
4934.08 & 0.00 & -0.150 & 8.042 & 0.88 \\
\hline & & & &
\end{tabular}

